\title{
SYMPLECTIC CUTS
}

\author{
EUGENE LERMAN
}

\begin{abstract}
According to McDuff the blow-up operation in symplectic geometry amounts to a removal of an open symplectic ball followed by a collapse of some boundary directions. In this paper I describe a generalization of the blow-up construction - the symplectic cut. In the case of symplectic manifolds with Hamiltonian circle action, the construction allows us to embed the reduced spaces in a symplectic manifold ("the symplectic cut") as codimension 2 symplectic submanifolds. Several applications are discussed.
\end{abstract}

\section{Introduction and the basic construction}

In the paper [GS5] on birational equivalence in the symplectic category, Guillemin and Sternberg observe a connection between reduction and blowups. The key idea can be seen in the following example. Consider $C \times C^{n}$ with coordinates $(w, z)=\left(w, z_{1}, \ldots, z_{n}\right)$ and with the standard symplectic form $\tau=-\sqrt{-1}\left(d w \wedge d \bar{w}+\sum d z_{j} \wedge d \bar{z}_{j}\right)$. Consider the Hamiltonian action of a circle $S^{1}$ on $C \times C^{n}$ given by

$$
e^{i \theta} \cdot(w, z)=\left(e^{-i \theta} w, e^{i \theta} z\right)
$$

with a momentum map

$$
\Phi(w, z)=\|z\|^{2}-|w|^{2} .
$$

Then the Marsden-Weinstein-Meyer reduced space at $\epsilon>0$ is the blow-up $\tilde{C}^{n}$ of the origin in $C^{n}$. Indeed, consider the map

$$
\begin{aligned}
\phi: C \times S^{2 n-1} & \rightarrow C \times C^{n} \\
(w, \zeta) & \mapsto\left(w,\left(\epsilon+|w|^{2}\right)^{1 / 2} \zeta\right),
\end{aligned}
$$

where $S^{2 n-1}=\left\{\zeta \in C^{n}:\|\zeta\|=1\right\}$. The map $\phi$ is an $S^{1}$ equivariant diffeomorphism onto the manifold $\Phi(w, z)=\epsilon$. Therefore,

$$
\Phi^{-1}(\epsilon) / S^{1}=\left(S^{2 n-1} \times C\right) / S^{1} .
$$

Received March 7, 1995.

Partially supported by an NSF postdoctoral fellowship. 
Also it is easy to see that

$$
\left.\phi^{*} \tau\right|_{\{0\} \times S^{2 n-1}}=\left.\frac{\epsilon}{\sqrt{-1}} \sum d \zeta_{j} \wedge d \bar{\zeta}_{j}\right|_{S^{2 n-1}},
$$

i.e., the restriction of the reduced symplectic form to the exceptional divisor is $\epsilon$ times the standard Fubini-Studi metric. Thus, the blow-up depends on a real parameter $\epsilon$. Guillemin and Sternberg call the reduced space $\Phi^{-1}(\epsilon) / S^{1}$ the blow-up of the origin in $C^{n}$ by an $\epsilon$ amount. Note also that if $\epsilon<0$, the reduced space is $C^{n}$.

There is another way to look at this construction. Namely, think of $\left(C^{n},-\sqrt{-1} \sum d z_{j} \wedge d \bar{z}_{j}\right)$ as a symplectic manifold $(M, \omega)$ with a Hamiltonian circle action and a momentum map $\mu(z)=\|z\|^{2}$. Then we have a Hamiltonian action of a circle $S^{1}$ on $\left(M \times C, \omega \oplus \frac{1}{\sqrt{-1}} d w \wedge d \bar{w}\right)$ given by $e^{i \theta} \cdot(m, w)=\left(e^{i \theta} m, e^{-i \theta} w\right)$ with a momentum map $\Phi(m, w)=\mu(m)-|w|^{2}$. The set $\{\Phi=\epsilon\}$ is a disjoint union of two $S^{1}$ invariant manifolds:

$$
\{\Phi=\epsilon\}=\begin{gathered}
\left\{(m, w): \mu(m)>\epsilon \quad \& \quad w=e^{i \theta} \sqrt{\mu(m)-\epsilon}\right\} \\
\bigsqcup \\
\{(m, 0): \mu(m)=\epsilon\} .
\end{gathered}
$$

The first manifold is equivariantly diffeomorphic to the product of $M_{\mu>\epsilon}:=$ $\{m \in M: \mu(m)>\epsilon\}$ and of the circle $S^{1}$ and the second manifold is diffeomorphic to the $\epsilon$ level set $\mu^{-1}(\epsilon)$. Consequently, the manifold $M_{\mu>\epsilon} \simeq$ $\left\{z \in C^{n}:\|z\|^{2}>\epsilon\right\}$ embeds into the reduced space $\{\Phi=\epsilon\} / S^{1}$ as an open dense symplectic submanifold and the remaining set, $\{\Phi=\epsilon\} / S^{1}-M_{\mu>\epsilon}$, is isomorphic to the reduced space $\mu^{-1}(\epsilon) / S^{1}$.

Thus, the $\epsilon$ blow-up of the origin in $C^{n}$ could be thought of as removing the ball of radius $\sqrt{\epsilon}$ centered at the origin and collapsing the fibers of the Hopf fibration in the boundary of the remaining set $\left\{z \in C^{n}:\|z\|^{2} \geq \epsilon\right\}$. This point of view is due to McDuff $[\mathrm{McD}]$.

1.1. Basic construction. Suppose now that $(M, \omega)$ is an arbitrary symplectic manifold with a Hamiltonian circle action and a momentum map $\mu: M \rightarrow R$. If the circle $S^{1}$ acts freely on a level set $\mu^{-1}(\epsilon)$, then $\epsilon$ is a regular value of the momentum map $\Phi(m, w)=\mu(m)-|w|^{2}$ arising from the action of $S^{1}$ on the product manifold $\left(M \times C, \omega \oplus \frac{1}{\sqrt{-1}} d w \wedge d \bar{w}\right)$, the action being $e^{i \theta} \cdot(m, w)=\left(e^{i \theta} m, e^{-i \theta} w\right)$. Then, as before, the manifold $M_{\mu>\epsilon}$ embeds as an open dense submanifold into the reduced space

$$
\bar{M}_{\mu \geq \epsilon}:=\Phi^{-1}(\epsilon) / S^{1}=\left\{(m, w) \in M \times C: \mu(m)-|w|^{2}=\epsilon\right\} / S^{1}
$$


and the difference $\bar{M}_{\mu \geq \epsilon}-M_{\mu>\epsilon}$ is symplectomorphic to the reduced space $\mu^{-1}(\epsilon) / S^{1}$. Topologically, $\bar{M}_{\mu \geq \epsilon}$ is the quotient of the manifold with boundary $M_{\mu \geq \epsilon}$ by the relation $\sim$ where $m \sim m^{\prime}$ if and only if $\mu(m)=\mu\left(m^{\prime}\right)=\epsilon$ and $m=e^{i \theta} m^{\prime}$ for some $e^{i \theta} \in S^{1}$.

A similar procedure defines

$$
\bar{M}_{\mu \leq \epsilon}=\left\{(m, w) \in M \times C: \mu(m)+|w|^{2}=\epsilon\right\} / S^{1} ;
$$

one only needs to look at the diagonal circle action on the product $(M \times$ $C, \omega \oplus \sqrt{-1} d w \wedge d \bar{w})$. The symplectic manifold $\mu^{-1}(\epsilon) / S^{1}$ is embedded in both $\bar{M}_{\mu \geq \epsilon}$ and $\bar{M}_{\mu \leq \epsilon}$ as a codimension 2 symplectic submanifold but with opposite normal bundles. So the symplectic gluing [G] of $\bar{M}_{\mu \geq \epsilon}$ and $\bar{M}_{\mu \leq \epsilon}$ along the reduced space $\mu^{-1}(\epsilon) / S^{1}$ recovers the original manifold $M$. For this reason, I would like to call the operation that produces $\bar{M}_{\mu \geq \epsilon}$ and $\bar{M}_{\mu \leq \epsilon}$ symplectic cutting. Note that there is no a priori reason to concentrate exclusively on $\bar{M}_{\mu \geq \epsilon}$; both sides of the cut may be interesting, depending on the context. For example, when $M=C^{n}, \bar{M}_{\mu \leq \epsilon}$ is isomorphic to the projective space $C P^{n}$ and the embedding of $M_{\mu<\epsilon}$ provides the projective space with a large Darboux chart. Perhaps one should think of this $C P^{n}$ as $C^{n}$ blown-up at infinity an $\infty-\epsilon$ amount.

Remark 1.1. (Kaehler structure on cuts.) If the manifold $(M, \omega)$ is Kaehler then its product with $C$ is Kaehler and so by theorem 3.5 of [GS2] the cut spaces $\bar{M}_{\mu \geq \epsilon}$ and $\bar{M}_{\mu \leq \epsilon}$, being the results of reduction, are also Kaehler. Note however that while the embedding $M_{\mu<\epsilon} \hookrightarrow \bar{M}_{\mu \leq \epsilon}$ is symplectic it is not a Kaehler isometry as the example of $M=\bar{C}^{n}$ clearly indicates.

Remark 1.2. (Cuts and Hamiltonian group actions.) If in addition to the action of a circle on our manifold $(M, \omega)$ we have a Hamiltonian action of another group $K$ on $M$ that commutes with the action of $S^{1}$ then the spaces $\bar{M}_{\mu \geq \epsilon}$ and $\bar{M}_{\mu \leq \epsilon}$ obtained by cutting $M$ along a level set $\mu^{-1}(\epsilon)$ are again Hamiltonian $K$ spaces. In particular, one always has a Hamiltonian circle action on the cut spaces.

Indeed, we can let $K$ act on the product $M \times C$ by simply letting it act on the first factor. Since this action of $K$ commutes with the two actions of $S^{1}$ of interest to us, it descends to a Hamiltonian action on the $S^{1}$-reduced spaces $\bar{M}_{\mu \geq \epsilon}$ and $\bar{M}_{\mu \leq \epsilon}$.

Remark 1.3. (Cuts and global blow-ups.) Let $(M, \omega)$ be a Hamiltonian $S^{1}$ space with a proper momentum map $\mu: M \rightarrow R$. Suppose the momentum map achieves its maximum on $M$ and that it achieves this maximal value $c$ at a single point $m_{0}$. Then for $\epsilon$ sufficiently small, $m_{0}$ is the only critical point in the set $M_{\mu>c-\epsilon}=\{m \in M: c-\mu(m)<\epsilon\}$. Assume 
further that the weights of the isotropy representation of $S^{1}$ on $T_{m_{0}} M$ are all 1. It follows from the equivariant Darboux theorem that the level sets $\mu=c-\delta$ are spheres for all $0<\delta<\epsilon$. Therefore, the manifold $\bar{M}_{\mu \leq c-\delta}$ is the blow-up of $M$ at $m_{0}$ by a $\delta$ amount since we obtained it by removing a set symplectomorphic to an open ball and then collapsing the fibers of the Hopf fibration in the boundary of the remaining set.

More generally, the momentum map may achieve its maximum on a symplectic submanifold $F$. Then, if the weights of the isotropy representation of $S^{1}$ on the normal bundle of $F$ are all 1 and $\delta$ is sufficiently small, the manifold $\bar{M}_{\mu \leq c-\delta}$ is the blow-up of $M$ along $F$ by a $\delta$ amount.

Remark 1.4. (Blow-up of $T^{*} S^{n}$ along the zero section.) Cuts may sometimes be used to blow up along a Lagrangian submanifold (a similar construction is described in $[\mathrm{G}]$ ).

Let $M$ be the cotangent bundle of the sphere $S^{n}$ with the standard symplectic form and the standard round metric $g$. Then the length function $\mu(q, p)=g_{q}(p, p)^{1 / 2}$, the square root of the energy, is smooth off the zero section of $T^{*} S^{n}$, so its Hamiltonian flow is well-defined. Note also that the flow has period 1 everywhere. Therefore we can construct

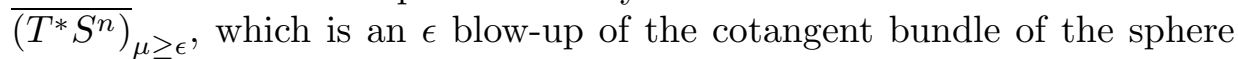
along the zero section. I must admit that the use of the word "blow-up" may be ill-advised at this point since there is no blow-down map. However, the inertia of using this word in the symplectic setting whenever one removes an open submanifold and then quotients the boundary along the null directions is too strong to resist.

Remark 1.5. (Cuts and moment polytopes.) Atiyah-Guillemin-Sternberg convexity theorem [A], [GS1] says that if a torus $T$ acts on a compact symplectic manifold $(M, \omega)$ with a moment map $\mu: M \rightarrow \mathfrak{t}^{*}$, then $\mu(M)$ is a rational convex polytope in $\mathfrak{t}^{*}$. We will refer to it as the moment polytope. Suppose $\xi \in \mathfrak{t}$ generates a circle subgroup $S_{\xi}$ of $T$. Then the action of $S_{\xi}$ on $(M, \omega)$ is Hamiltonian with moment map $\mu^{\xi}=\xi \circ \mu$. The actions of $S_{\xi}$ and of $T$ commute. If we cut $M$ at $\epsilon \in R$ using $\mu^{\xi}$, we get two Hamiltonian $T$ orbifolds $\bar{M}_{\mu \xi \geq \epsilon}$ and $\bar{M}_{\mu^{\xi} \leq \epsilon}$. Their moment polytopes are

$$
\mu(M) \cap\left\{\lambda \in \mathfrak{t}^{*}:\langle\xi, \lambda\rangle \geq \epsilon\right\} \text { and } \mu(M) \cap\left\{\lambda \in \mathfrak{t}^{*}:\langle\xi, \lambda\rangle \leq \epsilon\right\}
$$

respectively. Thus, there is a correspondence between symplectic cuts of manifolds and cuts of moment polytopes.

Delzant showed $[\mathrm{D}]$ that for effective completely integrable torus actions, the correspondence between actions and moment polytopes is one-to-one. 
An analogous theorem holds for orbifolds [LT]. Thus, for completely integrable torus actions, there is a one-to-one correspondence between symplectic cuts of spaces and partitions of polytopes by hyperplanes.

Remark 1.6. (Cuts and dynamical systems.) Suppose as before that we have a Hamiltonian circle action on a symplectic manifold $M$ with momentum map $\mu: M \rightarrow R$ and suppose that $h \in C^{\infty}(M)$ is an $S^{1}$ invariant Hamiltonian. Then $h$ defines an $S^{1}$ invariant function $\tilde{h}$ on $M \times C$ by $\tilde{h}(m, w)=h(m)$ and thereby, Hamiltonians $h_{\mu \leq \epsilon}$ and $h_{\mu \geq \epsilon}$ on $\bar{M}_{\mu \leq \epsilon}$ and $\bar{M}_{\mu \geq \epsilon}$, respectively. Note that the reduced dynamical system $\left(\mu^{-1}(\epsilon) / S^{1}, h_{\epsilon}\right)$ embeds into the cut systems

$$
\left(\bar{M}_{\mu \leq \epsilon}, h_{\mu \leq \epsilon}\right) \text { and }\left(\bar{M}_{\mu \geq \epsilon}, h_{\mu \geq \epsilon}\right) .
$$

Thus, one sees that the reduced dynamical system and parts of the original dynamical system can be combined into one system.

Remark 1.7. (Normal bundle of the reduced space in the cut spaces.) Since the normal bundle $\nu$ of the reduced space $\mu^{-1}(\epsilon) / S^{1}$ in the cut space $\bar{M}_{\mu \geq \epsilon}$ is given by

$$
\nu=\mu^{-1}(\epsilon) \times{ }_{S^{1}} C,
$$

the Euler class of $\nu$ is the Chern class of the principal $S^{1}$ bundle

$$
\mu^{-1}(\epsilon) \rightarrow \mu^{-1}(\epsilon) / S^{1}
$$

Remark 1.8. (Generalizations of the construction.) In conclusion we note that the basic construction of symplectic cutting can be generalized in several ways. First of all, a global Hamiltonian $S^{1}$ action is not necessary to define symplectic cuts. The only two properties of the level set $Z=\mu^{-1}(\epsilon)$ that we use are

- the difference $M-Z$ has two connected components and

- the null foliation of the pull-back $\left.\omega\right|_{Z}$ of the symplectic form to $Z$ is a principal circle fibration.

Thus, any hypersurface satisfying the two properties above allows us to carry out cuts.

Alternatively, we can drop the assumption that the action of the circle on the level set in question is free. If we keep the assumption that $\epsilon$ is a regular value of the moment map $\mu: M \rightarrow R$, then the action of the circle on the level set $\mu^{-1}(\epsilon)$ is locally free and so the cut spaces are orbifolds. This is the generic situation.

If we completely drop the regularity assumptions on the moment map then the reduced space $\mu^{-1}(\epsilon) / S^{1}$ and the cut spaces are symplectic stratified spaces (see $[\mathrm{SL}]$ ). 


\section{Applications}

In this section I describe several applications of symplectic cutting. These are a proof of the connectedness of fibers of moment maps arising from symplectic representations of compact groups (this will conclude the proof of the orbit conjecture of Benson et alii [BJLR]), a proof of Kirwan's convexity theorem, a proof of Kirwan's connectedness theorem and a proof of Kalkman's localization theorem.

Before giving any details, I would like to mention a very nice application of symplectic cutting that I will not describe here. This a proof of Duistermaat et alii [DGMW] in the case of circle actions of a conjecture of Guillemin and Sternberg that quantization commutes with reduction.

2.1. Connectedness of fibers of momentum maps for linear symplectic actions. A linear action of a compact Lie group $K$ on a symplectic vector space $(V, \omega)$ that preserves the symplectic form is necessarily Hamiltonian. The fibers of the corresponding momentum map $J: V \rightarrow \mathfrak{k}^{*}$ are intersections of real quadrics. These sets can be quite complicated and their connectedness is not at all apparent. F. Kirwan proved [K1] (see also [K2]) that if a momentum map arising from an action of a compact Lie group on a connected symplectic manifold is proper, then the preimages of coadjoint orbits under the map are connected. Consequently the fibers of the momentum map are connected. In our case the map $J$ need not be proper, so Kirwan's theorem doesn't apply directly. However, one can turn $J$ into a proper map by cutting away the infinity and thereby prove the following theorem.

Theorem 2.1. Let $K$ be a compact connected group acting linearly on a symplectic vector space $V$ and preserving its symplectic form $\omega$. Let $J$ : $V \rightarrow \mathfrak{k}^{*}$ denotes the corresponding moment map. Then for any coadjoint orbit $\mathcal{O}$ of $K$, the set $J^{-1}(\mathcal{O})$ is connected. Consequently, for any $\alpha \in \mathfrak{k}^{*}$, the fiber $J^{-1}(\alpha)$ is connected.

Proof. Without loss of generality, we may assume that $V$ is $C^{n}$ with the standard symplectic form and $K$ is a subgroup of the unitary group $U(n)$. Let $\mathcal{O}$ be a coadjoint orbit of $K$. We will show that for any $r>0$ the closed ball $\bar{B}(r)=\left\{z \in C^{n}:|z|^{2} \leq r\right\}$ intersects $J^{-1}(\mathcal{O})$ in a connected set. Clearly this will prove that $J^{-1}(\mathcal{O})$ is connected. Since $J^{-1}(\mathcal{O}) / K=$ $J^{-1}(\alpha) / K_{\alpha}$ for $\alpha \in \mathcal{O}$ and since the isotropy group $K_{\alpha}$ of $\alpha$ in $\mathfrak{k}^{*}$ is connected, the preimage of the orbit $\mathcal{O}$ is connected if and only if the reduced space $J^{-1}(\alpha) / K_{\alpha}$ is connected if and only if the fiber $J^{-1}(\alpha)$ is connected.

Now the fibers of the map $\pi: \bar{B}(r) \rightarrow \bar{C}^{n}|| z \|^{2} \leq r$, where $\left.\bar{C}^{n}|z|\right|^{2} \leq r$ is the blow-up of $C^{n}$ at infinity an $\infty-r$ amount, are points or circles. 
So a $e^{i \theta}$ invariant set $X$ in the closed ball is connected if and only its image under $\pi$ in the blow-up is connected. Since the action of $K$ on $C^{n}$ commutes with multiplication by $e^{i \theta}$, we see that the action of $K$ descends to a Hamiltonian action on the blow-up with momentum map $J_{r}:\left.\bar{C}^{n}|z|\right|^{2} \leq r \rightarrow \mathfrak{k}^{*}$ that makes the diagram

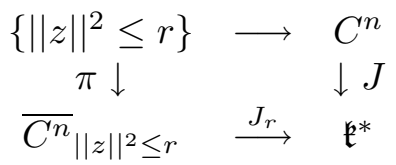

commute. Therefore, $J_{r}^{-1}(\mathcal{O})=\pi\left(J^{-1}(\mathcal{O}) \bigcap \bar{B}(r)\right)$. On the other hand, since the blow-up is the projective space $C P^{n}$, the momentum map $J_{r}$ is proper. So Kirwan's theorem (op. cit.) applies and the sets $J_{r}^{-1}(\mathcal{O})$ are connected. This, as we have seen, implies that the fibers of the original momentum map $J$ are connected as well and we are done.

2.2. Kirwan's convexity and connectedness theorems. It was observed by E. Meinrenken and C. Woodward that symplectic cuts can be used to reduce the proof of Kirwan's nonabelian convexity theorem to the convexity theorem for torus actions on orbifolds (an analog of AtiyahGuillemin-Sternberg convexity theorem for orbifolds was proved in [LT]). The cuts can also be used to reduce the proof of connectedness of fibers of a nonabelian moment map to the corresponding statement for abelian moment maps on orbifolds. We now sketch the arguments that should also work for actions on orbifolds. The details of the arguments will appear in [LMTW]. We start by giving precise statements of the theorems.

Theorem 2.2. Let $(M, \omega)$ be a compact connected symplectic manifold, $K \times(M, \omega) \rightarrow(M, \omega)$ a Hamiltonian action of a compact Lie group $K$ and $\mu: M \rightarrow \mathfrak{k}^{*}$ a corresponding equivariant moment map. Then the fibers of $\mu$ are connected.

Theorem 2.3. Let $(M, \omega), K$ and $\mu: M \rightarrow \mathfrak{k}^{*}$ be as in Theorem 2.2 above and let $\mathfrak{t}_{+}^{*} \subset \mathfrak{k}^{*}$ be a Weyl chamber. Then $\mu(M) \cap \mathfrak{t}_{+}^{*}$ is a rational convex polytope.

Theorem 2.2 is due to Kirwan [K1]. Theorem 2.3 is due to Guillemin and Sternberg in the Kaehler case [GS3] and to Kirwan in the general case [K2].

Sketch of proof. Assume for simplicity that the image of the moment map contains regular points in $\mathfrak{k}^{*}$ (i.e., points with abelian isotropy groups). More strongly, assume that generically in $M$ the isotropy groups are zero dimensional. The general case can be reduced to the one we are considering 
by means of the principal orbit type theorem. Fix a maximal torus $T$ of $K$, identify the dual of its Lie algebra $\mathfrak{t}^{*}$ with a subspace of $\mathfrak{k}^{*}$ and fix a Weyl chamber $\mathfrak{t}_{+}^{*}$. Consider the set $F=\mu^{-1}\left(\mathfrak{t}_{+}^{*}\right)$, the preimage of the interior of the Weyl chamber. Since the moment map $\mu$ is equivariant, it is transversal to $\mathfrak{t}_{+}^{*}$, hence $F$ is a $T$ invariant submanifold of $M$. By Theorem 26.7 of [GS4], $F$ is symplectic. It is not hard to see that $\mu_{F}:=\left.\mu\right|_{F}$ is a moment map for the symplectic action of $T$ on $F$.

One can show that $F$ is connected and that $K \cdot F$ is dense in $M$. Consequently the closure of the image of $F$ in $\mathfrak{t}^{*}$ is the intersection $\mu(M) \cap \mathfrak{t}_{+}^{*}$.

Since $F$ is not compact, $\mu_{F}$ is not proper. We now "compactify" $F$ as follows. Choose an affine hyperplane for each wall of the Weyl chamber, so that it is parallel to the wall, intersects the interior of the chamber and is distance less than $\epsilon \ll 1$ away from the wall. Cut the image $\mu_{F}(F)$ with these hyperlanes, i.e., cut $F$ using the actions of the circles corresponding to the simple roots (cf remark 1.5). The result for a generic choice of hyperplanes is a compact symplectic orbifold $\bar{F}_{\epsilon}$ which inherits a Hamiltonian action of the torus $T$ (cf proof of Theorem 2.1) and $\mu_{F}$ descends to a moment map $\bar{\mu}_{\epsilon}$. By the results of [LT], the fibers of $\bar{\mu}_{\epsilon}$ are connected and the image $\bar{\mu}_{\epsilon}\left(\bar{F}_{\epsilon}\right)$ is a rational convex polytope. Consequently, the fibers of $\mu_{F}$ are connected (cf again proof of Theorem 2.1) and $\mu_{F}(F)=\mu(F)$ is convex.

Since $\left.\mu\right|_{F}=\mu_{F}, \mu$ is equivariant and $K \cdot F$ is dense in $M$, it follows that, generically, the fibers of $\mu$ are connected. This is not quite enough to conclude that all the fibers of $\mu$ are connected. We need in addition the following topological fact.

Fact. Let $X$ be a manifold, $f: X \rightarrow V$ a vector space valued proper continuous map with the property that for any ball $B$ in $V$ there is a dense subset $U \subset B$ such that $f^{-1}(U)$ is connected. Then the fibers of $f$ are connected.

Since the set $K \cdot \mathfrak{\mathfrak { t }}_{+}^{*}$ is locally connected in $\mathfrak{k}^{*}$, we can now conclude that all the fibers of the moment map are connected.

To prove that the closure of $\mu(F)$ is a polytope, we need to make sure that the number of vertices of $\bar{\mu}_{\epsilon}\left(\bar{F}_{\epsilon}\right)$ is uniformly bounded as $\epsilon \rightarrow 0$. But the vertices lie in the intersections of the hyperlanes that we use for cutting with the singular values of the moment map $\mu_{T}$ arising from the Hamiltonian action of $T$ on $M$. By Atiyah-Guillemin-Sternberg convexity theorem, the singular values of $\mu_{T}$ are a finite union of convex polytopes. Consequently, the number of vertices of $\bar{\mu}_{\epsilon}\left(\bar{F}_{\epsilon}\right)$ is uniformly bounded and hence $\mu(M) \cap \mathfrak{t}_{+}^{*}$ is a convex polytope. 
2.3. A localization theorem. Consider a compact symplectic manifold $(M, \sigma)$ with a Hamiltonian action of a compact Lie group $K$ and let $\mu$ : $M \rightarrow \mathfrak{k}^{*}$ denote a corresponding moment map. Even when the group $K$ is a circle, it maybe difficult to understand the geometry of the corresponding Marsden-Weinstein-Meyer reduced spaces (cf. [GS2]). Therefore, one often considers a simpler problem-determining the cohomology rings of the reduced spaces. Kirwan proved in [K1] that the map $i^{*}: H_{K}^{\bullet}(M, C) \rightarrow$ $H_{K}^{\bullet}\left(\mu^{-1}(0), C\right)$ in equivariant cohomology induced by the inclusion $i$ : $\mu^{-1}(0) \rightarrow M$ is surjective. If the action of $K$ on $\mu^{-1}(0)$ is locally free, we then get a surjective ring homomorphism

$$
r: H_{K}^{\bullet}(M, C) \rightarrow H^{\bullet}\left(\mu^{-1}(0) / K, C\right)
$$

by composing $i^{*}$ with the isomorphism

$$
H_{K}^{\bullet}\left(\mu^{-1}(0), C\right) \simeq H^{\bullet}\left(\mu^{-1}(0) / K, C\right) .
$$

If we know the kernel of $r$, we can compute the cohomology ring of the reduced space $\mu^{-1}(0) / K$ in terms of the ring $H_{K}^{\bullet}(M, C)$. The map $r$ makes sense on the level of equivariant differential forms. A theorem of Jeffrey and Kirwan [JK] expresses the integral

$$
\int_{\mu^{-1}(0) / K} r(\alpha) e^{i \sigma_{0}}
$$

in terms of the restrictions of the equivariant form $\alpha \in \Omega_{K}^{\bullet}(M)$ to the fixed point set in $M$ of the maximal torus of $K$. Here and throughout the discussion we use the Cartan model of equivariant cohomology and we do not distinguish between cohomology classes and the forms that represent them. The symbol $\sigma_{0}$ denotes the reduced symplectic form on $\mu^{-1}(0) / K$. The paper $[\mathrm{JK}]$ is based on the results of Witten [W].

Independently of Jeffrey and Kirwan, Kalkman considered the case of $K=S^{1}$, a circle, [Ka]. By adapting Berline-Vergne localization theorem [BV] to manifolds with boundary, he showed that for an equivariant form $\alpha \in \Omega_{K}^{\bullet}(M)$ of degree $\operatorname{dim} M-1$, the integral

$$
\int_{\mu^{-1}(0) / K} r(\alpha)
$$

can be expressed in terms of the restrictions of $\alpha$ to certain components of the $K$ fixed point set $M^{K}$.

Symplectic cutting allows us to prove Kalkman's localization result directly from the Berline-Vergne localization. (This observation was also made independently by Siye $\mathrm{Wu}$ ). The proof consists of three steps. We first observe that there exists a ring map $s: H_{K}^{\bullet}(M) \rightarrow H_{K}^{\bullet}\left(\bar{M}_{\geq 0}\right)$ which preserves degrees and satisfies $i_{\mathcal{F}}^{*} s(\alpha)=i_{\mathcal{F}}^{*} \alpha$ for all $\mathcal{F} \subset M^{K}$ with 
$\mu(\mathcal{F})>0$ (where $i_{\mathcal{F}}: \mathcal{F} \rightarrow M$ denotes the embedding of $\mathcal{F}$ into $M$ ). To construct the map $s$, consider $H_{T^{2}}^{\bullet}(M \times C)$, where $T^{2}=K \times S^{1}$ acts on $M \times C$ by $(k, t) \cdot(m, w)=\left(k \cdot m, t^{-1} w\right)$. This cohomology ring is isomorphic to $H_{K}^{\bullet}(M) \otimes H_{S^{1}}^{\bullet}(C)=H_{K}^{\bullet}(M) \otimes C[y]$. Therefore, $H_{K}^{\bullet}(M)$ injects into $H_{T^{2}}^{\bullet}(M \times C)$. On the other hand, we have a $T^{2}$ equivariant inclusion

$$
i:\left\{(m, w): \mu(m)-|w|^{2}=0\right\} \hookrightarrow M \times C .
$$

Composing $H_{K}^{\bullet}(M) \hookrightarrow H_{T^{2}}^{\bullet}(M \times C)$ with the pull-back $i^{*}: H_{T^{2}}^{\bullet}(M \times C) \rightarrow$ $H_{T^{2}}^{\bullet}\left(\left\{\mu(m)=|w|^{2}\right\}\right)$, we get a map $H_{K}^{\bullet}(M) \rightarrow H_{T^{2}}^{\bullet}\left(\left\{\mu(m)=|w|^{2}\right\}\right)$. It remains to observe that since the action of the diagonal circle $\Delta$ in $T^{2}$ is locally free on $\left\{\mu(m)=|w|^{2}\right\}$, we have $H_{T^{2}}^{\bullet}\left(\left\{\mu(m)=|w|^{2}\right\}\right)=$ $H_{T^{2} / \Delta}^{\bullet}\left(\left\{\mu(m)=|w|^{2}\right\} / \Delta\right)$. The quotient $\left\{\mu(m)=|w|^{2}\right\} / \Delta$ is the cut space $\bar{M}_{\mu \geq 0}$ and $H_{T^{2} / \Delta}^{\bullet}=H_{K}^{\bullet}$ since $T^{2} \simeq K \times \Delta$. Therefore, we get a $\operatorname{map} s: H_{K}^{\bullet}(M) \rightarrow H_{K}^{\bullet}\left(\bar{M}_{\mu \geq 0}\right)$.

The second step is to apply Berline-Vergne localization on the cut space $\bar{M}_{\mu \geq 0}$ to $s(\alpha)$ for $\alpha \in \Omega_{S^{1}}^{\bullet}(M)$ of degree $\operatorname{dim} M-2$. Using the fact that $i_{\mathcal{F}}^{*} s(\alpha)=i_{\mathcal{F}}^{*} \alpha$ we then get

$$
\int_{M_{0}} \frac{i_{M_{0}}^{*} s(\alpha)}{e\left(M_{0}\right)}+\sum_{\mathcal{F}, \mu(\mathcal{F})>0} \int_{\mathcal{F}} \frac{i_{\mathcal{F}}^{*} \alpha}{e(\mathcal{F})}=0
$$

Here $M_{0}$ is the reduced space $\mu^{-1}(0) / K, e\left(M_{0}\right)$ is the equivariant Euler class of its normal bundle in $\bar{M}_{\mu \geq 0}$, the $\mathcal{F}$ 's are connected components of $M^{S^{1}}$ and the $e(\mathcal{F})$ 's are the corresponding equivariant Euler classes.

Note that the integrals $\int_{\mathcal{F}} i_{\mathcal{F}}^{*} \alpha / e(\mathcal{F})$ are rational functions in one indeterminate $x$. Therefore, it makes sense to take residues, that is to say, the coefficients of $x^{-1}$ in the integrals. Equation (1) becomes

$$
-\left.r e s\right|_{x=0} \int_{M_{0}} \frac{i_{M_{0}}^{*} s(\alpha)}{e\left(M_{0}\right)}=\left.\sum_{\mathcal{F}, \mu(\mathcal{F})>0} r e s\right|_{x=0} \int_{\mathcal{F}} \frac{i_{\mathcal{F}}^{*} \alpha}{e(\mathcal{F})} .
$$

The third step is an observation that

$$
-\left.r e s\right|_{x=0} \int_{M_{0}} \frac{i_{M_{0}}^{*} s(\alpha)}{e\left(M_{0}\right)}=\int_{M_{0}} r(\alpha)
$$

combined with the fact that the Euler class of the normal bundle of $M_{0}$ in $\bar{M}_{\mu \geq 0}$ is the Chern class of the circle bundle $\mu^{-1}(0) \rightarrow M_{0}$ (see Remark 1.7). A detailed discussion of this step can be found in [GK]. The key fact is that, on the level of forms, the map $H_{K}^{\bullet}\left(\mu^{-1}(0), C\right) \rightarrow$ $H^{\bullet}\left(\mu^{-1}(0) / K, C\right)$ is given by substituting the curvature for the indeterminate $x$. 


\section{Acknowledgments}

I would like to thank Siye Wu, Chris Woodward and Victor Guillemin for encouraging me to write down these observations. I would also like to thank Reyer Sjamaar and Yael Karshon for commenting on an earlier version of the manuscript.

\section{References}

[A] M. F. Atiyah, Convexity and commuting hamiltonians, Bull. London Math. Soc. 14 (1982), 1-15.

[BJLR] C. Benson, J. Jenkins, R. L. Lipsman and G. Ratcliff, The moment map for a multiplicity free action, Bull. AMS 31 (1994), 185-189.

[BV] N. Berline and M. Vergne, Classes caractéristiques équivariantes, C. R. Acad. Sci. Paris 295 (1982) 539-541.

[D] T. Delzant, Hamiltoniens périodiques et images convexes de l'application moment, Bull. Math. France 116 (1988), 315-339.

[DGMW] J. J. Duistermaat, V. Guillemin, E. Meinrenken and S. Wu, Symplectic reduction and Riemann-Roch for circle actions, Math. Research Letters, 2 (1995) $259-266$.

[G] R. Gompf, A new construction of symplectic manifolds, Ann. Math., to appear.

[GK] V. Guillemin and J. Kalkman, A new proof the Jeffrey-Kirwan localization theorem, MIT preprint, (September 1994).

[GS1] V. Guillemin and S. Sternberg, Convexity properties of the moment mapping I, Invent. Math. 67 (1982), 491-513.

[GS2] - Geometric quantization and multiplicities of group representations, Invent. Math. 67 (1982), 515-538.

[GS3] _ Convexity properties of the moment mapping II, Invent. Math. 77 (1984), 533-546.

[GS4] , Symplectic techniques in physics, Cambridge Univ. Press, 1984.

[GS5] _ Birational equivalence in the symplectic category, Invent. Math. 97 (1989), 485-522.

[JK] L. Jeffrey and F. Kirwan, Localization for non-abelian group actions, alggeom/9307001, to appear in Topology.

[Ka] J. Kalkman, Cohomology rings of symplectic quotients, J. Reine Angew. Math., 458 (1995), 37-52.

[K1] F. Kirwan, Cohomology of quotients in symplectic and algebraic geometry, Mathematical Notes 31, Princeton University Press, Princeton, 1984.

[K2] _ Convexity properties of the moment mapping III, Invent. Math. 77 (1984), 547-552.

[LMS] E. Lerman, R. Montgomery and R. Sjamaar, Examples of singular reduction, in Symplectic geometry, D. A. Salamon, ed., Cambridge: Cambridge University Press, 1993. 
[LT] E. Lerman and S. Tolman, Symplectic toric orbifolds, dg-ga/9412005.

[LMTW] E. Lerman, E. Meinrenken, S. Tolman and C. Woodward, Nonabelian convexity via symplectic cuts, in preparation.

[McD] D. McDuff, Blow-ups and symplectic embeddings in dimension 4, Topology 30 (1991), 409-421.

[SL] S. Sjamaar and E. Lerman, Stratified symplectic spaces and reduction, Ann. Math. 134 (1991), 375-422.

[W] E. Witten, Two dimensional gauge theories revisited, J. Geom. Phys. 9 (1992), 303-368.

Department of Mathematics, Mit, Cambridge, MA 02139

E-mail address: eugene@math.mit.edu 